\title{
On approximations involved in the theory of positron transport in gases in electric and magnetic fields
}

\author{
A. Banković ${ }^{1, a}$, S. Dujko ${ }^{1,2}$, R.D. White ${ }^{3}$, S.J. Buckman ${ }^{4}$, and Z.Lj. Petrović ${ }^{1}$ \\ 1 Institute of Physics, University of Belgrade, P.O. Box 68, Pregrevica 118, 11080 Zemun, Belgrade, Serbia \\ 2 Centrum Wiskunde and Informatica (CWI), P.O. Box 94079, 1090 GB Amsterdam, The Netherlands \\ 3 ARC Centre for Antimatter-Matter Studies, School of Engineering and Physical Sciences, James Cook University, \\ 4810 Townsville, Australia \\ 4 ARC Centre for Antimatter-Matter Studies, Australian National University, ACT 0200 Canberra, Australia
}

Received 30 November 2011 / Received in final form 13 April 2012

Published online 10 July 2012 - (c) EDP Sciences, Società Italiana di Fisica, Springer-Verlag 2012

\begin{abstract}
A multi term theory for solving Boltzmann's equation is briefly reviewed and used to test various concepts and approximate expressions for the determination of the positron transport properties in neutral molecular gases in crossed electric and magnetic fields. Among many important approximations which have found their way into contemporary positron studies, the following are particularly discussed: (1) is the approximation of using the cross sections for the electron scattering to describe the positron behavior satisfactory, (2) how accurate is two term approximation for solving Boltzmann's equation in the context of positron studies, and (3) what is the domain of applicability of Langevin elementary transport theory and Tonks' theorem for positrons in electric and magnetic fields. We highlight the limitations, range of applicability and inadequacies of such assumptions for positrons in $\mathrm{H}_{2}$ and $\mathrm{N}_{2}$. It is pointed out that there is no real alternative to the accurate multi term theory and/or Monte Carlo simulations if high precision is required. It is demonstrated that if the demands for accuracy associated with some of these approximations are relaxed, results may not be even qualitatively correct.
\end{abstract}

\section{Introduction}

The theoretical investigation of positron swarms moving in an unbounded gas in electric and magnetic fields is a topic of great interest both as a problem in basic physics and for its potential for application to modern technology [1-3]. Transport processes of a swarm of positrons are related to various problems in different areas, ranging from astrophysics [4] and material science (see for example [5] and references therein) to medicine [6]. While the understanding of the interaction of high energy positrons with soft, biological matter is of key importance for medical diagnostic procedures, such as positron emission tomography $[7,8]$, the knowledge of low-energy positron transport in gases under the influence of electric and magnetic fields plays a crucial role in optimizing the positron traps. These traps are usually operated under various configurations of electric and magnetic fields with specific requirements on the transport properties of the positrons needed to achieve the desired resolution of high-flux low-energy positron beams $[9,10]$. Additional issues, like those relevant for the collision-dominated cooling stage of PenningMalmberg-Surko trap [11,12]: (1) how to minimize losses due to Ps formation; and (2) what is the most suitable

\footnotetext{
${ }^{a}$ e-mail: ana.bankovic@gmail.com
}

buffer gas composition; or those relevant for the dumping stage of the trap: (1) what are the most important temporal and energy effects; and (2) how does changing potentials affect the dropping positrons out of the trap; should dominantly be a subject of swarm studies.

Essentially, there are two major techniques which can be used to analyze the positron transport in gases in varying configurations of electric and magnetic fields, the Boltzmann equation analysis $[13,14]$ and Monte Carlo simulation [15-19]. One ought to mention the Monte Carlo codes that have been specifically developed to model positron emission tomography diagnostic technique [20-24] and those developed to model tracks of particles in water [25]. However the mathematical complexity associated with accurate multi term solutions of the Boltzmann equation and/or computation and memory costs due to the statistical nature of Monte Carlo methods, limit their efficient use in modeling the positron transport, especially if systems in real geometries are considered. As is now common place in the field of low-temperature plasma discharges, fluid equation approaches represent a computational efficient and flexible means to model such complex systems $[26,27]$. Rather than solving for the phase space distribution directly through Boltzmann's equation, fluid equation approaches solve for low-order velocity moments of the distribution function through balance 
equations for particle number, momentum and energy, depending of the level of approximation. To enhance the accuracy of such models, accurate transport data (e.g. drift and diffusion coefficients) with consistent implementation are generally required input data [27]. For complex geometries involving electric and magnetic fields, empirical transport data is generally not available and one often resorts to approximate relations (e.g. Langevin theories [28,29], Tonks' theorem [30-33] and equivalent field approximations [34]) in order to furnish such data. The accuracy of such approximate treatments for positrons is the focus of the current paper.

We begin this paper with a brief overview of swarm transport theory including a multi term solution of the Boltzmann equation when both the electric and magnetic fields are present and when non-conservative collisions are operative. In Section 3.2, we apply this technique to positrons and electrons in $\mathrm{H}_{2}$ and $\mathrm{N}_{2}$ where we highlight the differences between the transport properties for these two charged particle species. Comparisons are made with the goal of testing the approximation of using the cross sections for the electron scattering to describe the positron transport in neutral gases. The next important aspect of the present paper concerns the adequacy of the two term theory for positrons. In Section 3.3, the two term approximation is tested for positrons in $\mathrm{N}_{2}$, as a typical representative of molecular gases. More importantly, $\mathrm{N}_{2}$ is often employed as a buffer gas in positron traps and hence it is of great importance to check the domain of validity of the two term theory for this gaseous medium. In Sections 3.4 and 3.5, we then give a brief discussion concerning the accuracy and range of applicability of the Langevin's elementary transport theory and Tonks' theorem, respectively, for positrons in an electric field only situation and in crossed electric and magnetic fields. In particular, the main advantage of Tonks' theorem is that one can determine general transport properties, for example the mean energy, drift speed and rate coefficients for different collisional processes in terms of an electric field only data. We will illustrate this approach and its limitations in Section 3.5 for positrons in $\mathrm{N}_{2}$ and $\mathrm{H}_{2}$.

\section{Transport theory}

The governing equation describing a swarm of charged particles moving through a background neutral gas under the influence of electric and magnetic fields is given by Boltzmann's equation for the phase-space distribution function $f(\boldsymbol{r}, \boldsymbol{c}, t)$ :

$$
\frac{\partial f}{\partial t}+\boldsymbol{c} \cdot \frac{\partial f}{\partial \boldsymbol{r}}+\frac{q}{m}[\boldsymbol{E}+\boldsymbol{c} \times \boldsymbol{B}] \cdot \frac{\partial f}{\partial \boldsymbol{c}}=-J\left(f, f_{0}\right) .
$$

Here $r$ and $c$ denote the charged particle and velocity coordinates, $q$ and $m$ are the charge and mass of the swarm particle, $t$ is time while $E$ and $B$ are magnitudes of the electric and magnetic fields, respectively. In what follows, we employ a co-ordinate system in which $\boldsymbol{E}$ defines the $z$-axis while $\boldsymbol{B}$ lies in the $y$ - $z$ plane, making an angle $\psi$ with respect to the $\boldsymbol{E}$. The right-hand side of equation (1) denotes the linear charged particle-neutral molecule collision operator, accounting for elastic, inelastic and nonconservative (the collisions which changes the number of the particles within the swarm, e.g. Ps formation and annihilation for positrons and dissociative attachment and ionization for electrons) collisions. For elastic collisions we use the original Boltzmann collision operator [35], while for inelastic collisions we prefer the semiclassical generalization of Wang-Chang et al. [36]. The collision operators for non-conservative collisions are discussed in $[37,38]$. It should be noted that the collision operator for Ps formation and/or positron annihilation for positrons has the same form as the attachment operator for electrons.

Swarm conditions are assumed to apply where the charged particle number density is much less than number density of neutral species and mutual interactions between charged particles are negligible compared to the charged particle-neutral particle interactions. We assume the neutral molecules (of number density $n_{0}$ ) remain in thermal equilibrium at a temperature $T_{0}$ and their internal states are characterized by a Boltzmann distribution.

The methods and techniques for solving Boltzmann's equation are by now standard and the reader is referred to recent reviews for details $[13,14,37,39]$. Among many important aspects, we emphasize the following important steps in solving Boltzmann's equation:

(1) The angular dependence of the phase-space distribution function in velocity space can be represented in terms of an expansion in spherical harmonics:

$$
f(\boldsymbol{r}, \boldsymbol{c}, t)=\sum_{l=0}^{\infty} \sum_{m=-l}^{l} f_{m}^{(l)}(\boldsymbol{r}, c, t) Y_{m}^{[l]}(\hat{\boldsymbol{c}}),
$$

where $Y_{m}^{[l]}(\hat{\boldsymbol{c}})$ are spherical harmonics and $\hat{\boldsymbol{c}}$ denotes the angles of $\boldsymbol{c}$. The value of $l$ is incremented until some predefined accuracy criterion is satisfied. This value indicates the deviation of the velocity distribution function from isotropy. The classical two term approximation often used for the determination of electron transport properties in plasma modeling community, is based upon the choice of setting the upper bound on the summation in $(2)$ to $l_{\max }=1$. In what follows, the validity of two term approximation for positrons in molecular gases will be investigated.

(2) The speed (energy) dependence of the coefficients in equation (2) is represented by an expansion about a Maxwellian at an arbitrary temperature $T_{b}(t)$, in terms of Sonine polynomials. Other options are available to resolve the speed dependence of $f(\boldsymbol{r}, \boldsymbol{c}, t)$, for example the finite-difference schemes, polynomial expansions or pseudo-spectral methods.

(3) Under the hydrodynamic conditions, the spatial dependence of $f(\boldsymbol{r}, \boldsymbol{c}, t)$ is treated by the density gradient expansion [40]:

$$
f(\boldsymbol{r}, \boldsymbol{c}, t)=\sum_{k=0}^{\infty} f^{(k)}(\boldsymbol{c}, t) \odot(-\nabla)^{k} n(\boldsymbol{r}, t),
$$


where $f^{(k)}(\boldsymbol{c}, t)$ are tensors of rank $k$ and $\odot$ denotes a $k$-fold scalar product.

(4) Using the appropriate orthogonality relations for the spherical harmonics and modified Sonine polynomials, Boltzmann's equation is converted into a hierarchy of coupled differential equations for the moments of the distribution function. Discretising in time using an implicit finite difference scheme converts the system of coupled differential equations into a hierarchy of coupled matrix equations. These equations are numerically solved and all the transport properties are expressed in terms of the moments of the distribution function [14].

\subsection{The duality of transport coefficients}

The connection between experimental and theoretical investigations of charged particle transport behavior is made through the equation of continuity

$$
\frac{\partial n(\boldsymbol{r}, t)}{\partial t}+\nabla \cdot \boldsymbol{\Gamma}(\boldsymbol{r}, t)=S(\boldsymbol{r}, t)
$$

where

$$
n(\boldsymbol{r}, t)=\int f(\boldsymbol{r}, \boldsymbol{c}, t) d t
$$

while $\boldsymbol{\Gamma}(\boldsymbol{r}, t)=n\langle\boldsymbol{c}\rangle$ is the charged particle flux and $S(\boldsymbol{r}, t)$ represents the production rate per unit volume per unit time arising from non-conservative collisional processes. Assuming the functional relationship (3) the flux and source term could be also expanded in terms of powers of the density gradients:

$$
\begin{aligned}
\boldsymbol{\Gamma}(\boldsymbol{r}, t)= & \boldsymbol{W}^{\star}(t) n(\boldsymbol{r}, t)-\boldsymbol{D}^{\star}(t) \cdot \nabla n(\boldsymbol{r}, t)+\ldots, \\
S(\boldsymbol{r}, t)= & S^{(0)}(t) n(\boldsymbol{r}, t)-\boldsymbol{S}^{(1)}(t) \cdot \nabla n(\boldsymbol{r}, t)+\boldsymbol{S}^{(2)}(t) \\
& : \nabla \nabla n(\boldsymbol{r}, t)+\ldots,
\end{aligned}
$$

where $\boldsymbol{W}^{\star}(t)$ and $\boldsymbol{D}^{\star}(t)$ define, respectively, the flux drift velocity and flux diffusion tensor. Substitutions of expansions (6) and (7) into the continuity equation (4) results in truncation the second order in the density gradients, the diffusion equation:

$$
\frac{\partial n}{\partial t}+\boldsymbol{W}(t) \cdot \nabla n-\boldsymbol{D}(t): \nabla \nabla n=-R_{a}(t) n,
$$

where $R_{a}(t), \boldsymbol{W}(\mathrm{t})$ and $\boldsymbol{D}(\mathrm{t})$ are, respectively, the loss rate coefficient, bulk drift velocity, and the bulk diffusion tensor. These transport properties contain explicit contributions from non-conservative collisions and are given by

$$
\begin{aligned}
R_{a}(t) & =S^{(0)}(t)-\int J^{R}\left[f^{(0)}(\boldsymbol{c}, t)\right] d \boldsymbol{c}, \\
\boldsymbol{W}(t) & =\boldsymbol{W}^{\star}(t)-\boldsymbol{S}^{(1)}(t) \\
& =\int \boldsymbol{c}\left[f^{(0)}(\boldsymbol{c}, t)\right] d \boldsymbol{c}-\int J^{R}\left[f^{(1)}(\boldsymbol{c}, t)\right] d \boldsymbol{c} \\
\boldsymbol{D}(t) & =\boldsymbol{D}^{\star}(t)-\boldsymbol{S}^{(2)}(t) \\
& =\int \boldsymbol{c}\left[f^{(1)}(\boldsymbol{c}, t)\right] d \boldsymbol{c}-\int J^{R}\left[f^{(2)}(\boldsymbol{c}, t)\right] d \boldsymbol{c},
\end{aligned}
$$

where $J^{R}$ denotes the reactive part of the collision operator.

It is clear that one must be cautious when considering the nature of the transport data. Firstly, the second order density gradient expansion of the phase-space distribution function is necessary to determine the explicit contribution of non-conservative collisions to diffusion coefficients. Secondly, the bulk transport coefficients contain both the implicit (i.e., the effects of non-conservative collisions on the velocity distribution function only) and explicit effects of non-conservative collisions while the flux properties contain only the implicit effects. For electrons, the distinction between these two sets of transport coefficients was discussed at length in the 1980s, but has generally been ignored in the majority of previous work in the plasma modeling community. This has lead to a potentially serious mismatch between input swarm data (generally the bulk transport properties) and the parameters (often the flux transport properties) required in many plasma fluid models [27]. Experimentally, there is no strategy how to resolve the distinction between these two sets of data and only theory, i.e. Boltzmann's equation calculations and/or Monte Carlo simulations, can resolve any such mismatch, by providing both flux and bulk transport coefficients. The results in Section 3 highlight the often large differences in the magnitudes and profiles between the two sets of transport coefficients.

\section{Results and discussion}

\subsection{Preliminaries}

As discussed in Section 1, the aim of this work is to test various approximate formulas for the determination of positron transport properties in gases when both the electric and magnetic fields are present against a highly accurate multi term theory for solving Boltzmann's equation. We consider the reduced electric field $E / n_{0}$ range: 0.1-100 Td $\left(1 \mathrm{Td}=10^{-21} \mathrm{Vm}^{2}\right)$ and the reduced magnetic field $B / n_{0}$ range: $0-2000 \mathrm{Hx}\left(1 \mathrm{Hx}=10^{-27} \mathrm{~T} \mathrm{~m}^{-3}\right)$. The cross sections for the electron scattering in $\mathrm{N}_{2}$ detailed by Stojanović and Petrović [41], and cross sections for electron scattering in $\mathrm{H}_{2}$ developed by Yoon et al. [42] are implemented in this work. These two sets include cross sections for momentum transfer in elastic collisions, ionization, electron attachment, electronic, vibrational and rotational excitations. Both of these sets are tested using a standard swarm analysis [43] and a good agreement between calculated transport parameters and those measured in experiments have been found. For positron scattering in $\mathrm{N}_{2}$ and $\mathrm{H}_{2}$, we have used cross sections compiled by Banković et al. [44,45]. These data include the cross sections for elastic collisions, Ps formation, direct ionization, electronic and vibrational excitations. The individual cross sections for positrons have their experimental uncertainty as given in the original papers but it was not possible to test the collective accuracy for the set due to lack of experimental positron swarm transport data. Yet this set is the best that is available at the moment and 


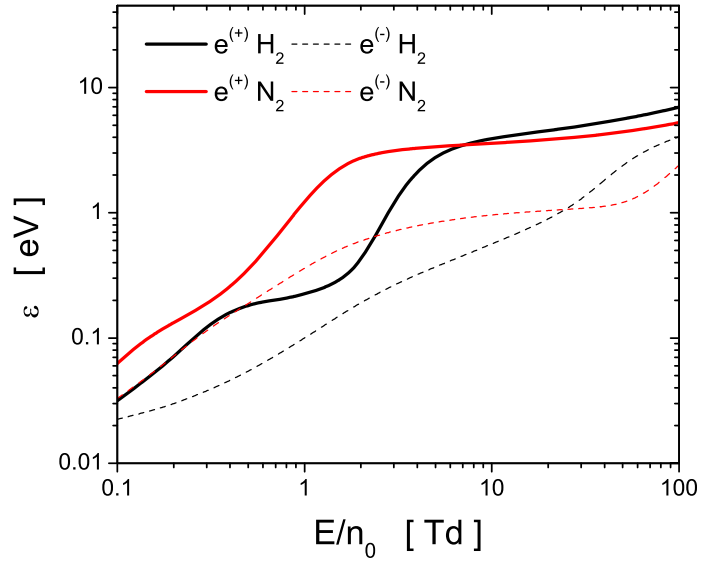

Fig. 1. (Color online) Variation of the mean energy with $E / n_{0}$ for electrons and positrons in $\mathrm{H}_{2}$ and $\mathrm{N}_{2}$.

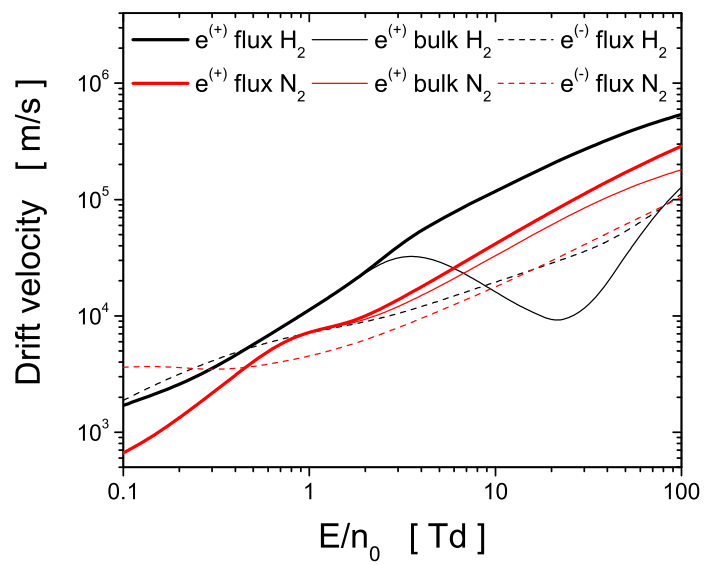

Fig. 2. (Color online) Variation of the bulk and flux drift velocity components with $E / n_{0}$ for electrons and positrons in $\mathrm{H}_{2}$ and $\mathrm{N}_{2}$.

can provide a good qualitative representation of positron transport, for example the effect of large non-conservative positronium formation cross section. The gas number density is $3.54 \times 10^{22} \mathrm{~m}^{-3}$ which corresponds to the pressure of 1 Torr at $273 \mathrm{~K}$.

\subsection{Is the approximation of using the cross sections for the electron scattering to describe the positron behavior satisfactory?}

In Figures 1-4 we show the variation of the mean energy, drift velocity and diffusion coefficients, respectively, with the reduced electric field $E / n_{0}$ for electrons and positrons in $\mathrm{H}_{2}$ and $\mathrm{N}_{2}$. The properties of the cross sections are reflected in the profiles of the mean energies for both the electrons and positrons. Comparing mean energies for electrons and positrons, it is evident that the mean positron energies dominate the mean electron energies. In particular, for an intermediate range of $E / n_{0}$ covered in this work, we see that the differences between the mean energies for electrons and positrons in $\mathrm{H}_{2}$ can be up to an order of magnitude.

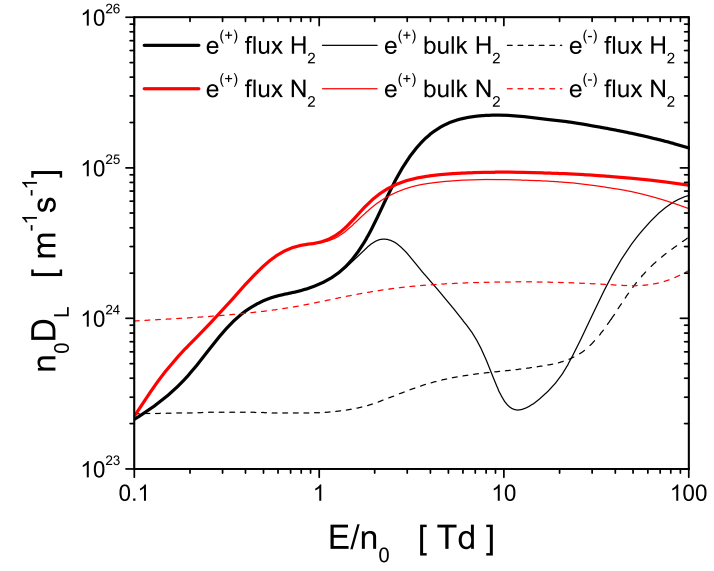

Fig. 3. (Color online) Variation of the bulk and flux longitudinal diffusion coefficients with $E / n_{0}$ for electrons and positrons in $\mathrm{H}_{2}$ and $\mathrm{N}_{2}$.

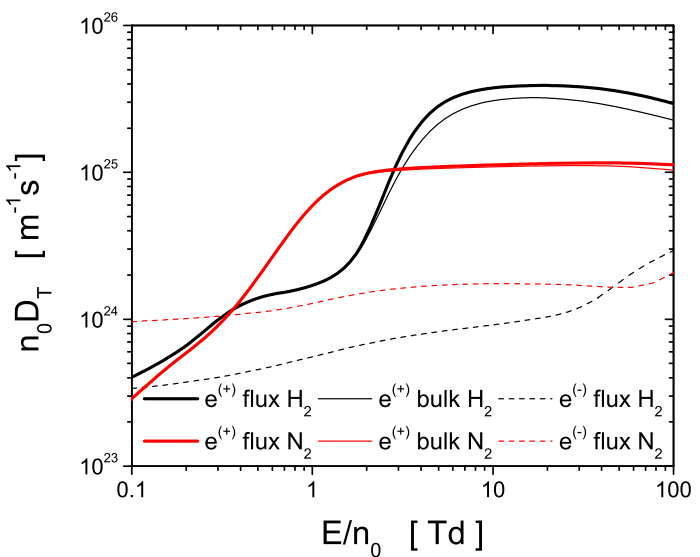

Fig. 4. (Color online) Variation of the bulk and flux transverse diffusion coefficients with $E / n_{0}$ for electrons and positrons in $\mathrm{H}_{2}$ and $\mathrm{N}_{2}$.

From the profiles of the drift velocity components for electrons in $\mathrm{H}_{2}$ and $\mathrm{N}_{2}$ and for positrons in $\mathrm{N}_{2}$ shown in Figure 2, we see that there are no signs of a negative differential conductivity (NDC) effect, i.e., both the bulk and flux drift velocity components are monotonically increasing functions of $E / n_{0}$. For clarity, only the flux drift velocity component is shown for electrons as there are no significant differences between the bulk and flux components over a range of $E / n_{0}$ considered in this work. However, from the plot of $W$ for positrons in $\mathrm{H}_{2}$ we see that positrons exhibit a very pronounced NDC, i.e., over a range of $E / n_{0}$ values the drift velocity decreases as the field is increased. In contrast to bulk component, and in contrast to anything previously observed for electrons, there are no signs of NDC in the flux component. As a consequence, the differences between the bulk and flux components are greater than an order of magnitude for certain values of $E / n_{0}$. Conditions leading to this phenomenon for positrons has been recently discussed by Banković et al. [45-47] and even experimental observations have been reported $[48,49]$. 
Figures 3 and 4 show the longitudinal and transverse diffusion coefficients as a function of $E / n_{0}$, respectively, for electrons and positrons in $\mathrm{H}_{2}$ and $\mathrm{N}_{2}$. The explicit effects of ionization on an electron diffusion are weak in the energy range considered in this work and hence only the flux components of the diffusion coefficients for electrons are shown. However, the explicit contribution of Ps formation to the measurable diffusion coefficients for positrons in $\mathrm{N}_{2}$, and particularly in $\mathrm{H}_{2}$ is clearly demonstrated. We see that the bulk components are greater than their corresponding flux components indicating that the decrease in positron numbers due to Ps formation reduces diffusion in both parallel and perpendicular directions. The difference of almost two orders of magnitude between the flux and bulk longitudinal diffusion coefficients for positrons in $\mathrm{H}_{2}$ is another dramatic manifestation of positron transport modification induced by the explicit influence of Ps formation. Such a huge difference between the flux and bulk diffusion components has never been observed for electrons even under extreme conditions which may lead to the development of absolute negative electron mobility $[50,51]$.

These examples demonstrate how important it is to employ the accurate cross sections for positron scattering in applications involving positrons. Approximations based on using the cross-sections for the electron scattering to describe positron behavior can seriously plague the modeling as results may not be even qualitatively correct.

\subsection{How accurate is the two term approximation for solving Boltzmann's equation for positrons in molecular gases?}

In this section we investigate the accuracy of the two term approximation for solving Boltzmann's equation for positrons in gases. In other words, the aim of this section is to study the convergence trend in the $l$-index as a function of $E / n_{0}$. For illustrative purpose, we restrict our discussion to positrons in $\mathrm{N}_{2}$. In Figure 5 we display the percentage difference between the two term and fully converged results. Truncation $l_{\max }=5$ was required to achieve $1 \%$ accuracy for all transport coefficients and properties.

Errors associated with the two term approximation are clearly evident for all transport properties. We observe that different transport coefficients show different convergence trends and have different rates of convergence. For a chosen set of conditions, the mean energy and rate coefficient for Ps formation have the errors of the order of $20 \%$ while the longitudinal diffusion coefficient has the errors of the order of $30 \%$. The most dramatic situation exists for the transverse diffusion coefficient where the errors are of the order of $250 \%$ in the field range considered. We observe that increasing $E / n_{0}$ deteriorates the accuracy of the two term approximation, although this is not always clear as evidenced by the trend of the bulk longitudinal diffusion coefficient. Intuitively we expect this, since for an increasing $E / n_{0}$ the inelastic collisions start to play a more significant role and an enhancement in the asymme-

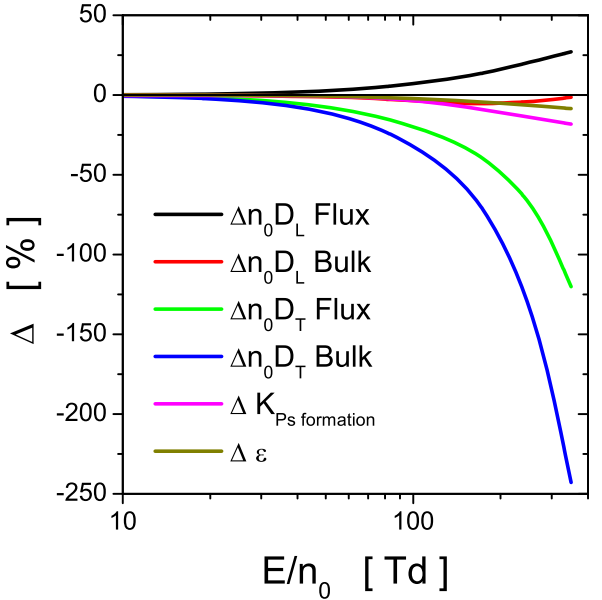

Fig. 5. (Color online) Percentage difference between the two term and multi term results for various transport properties for positrons in $\mathrm{N}_{2}$.

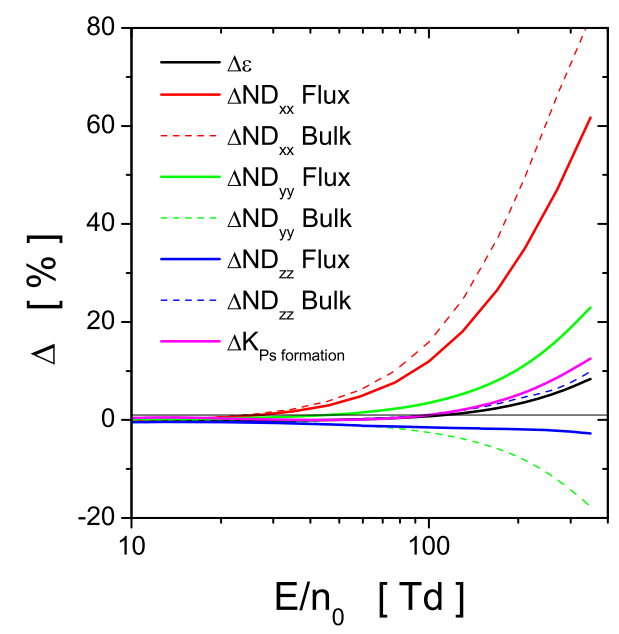

Fig. 6. (Color online) Percentage difference between the two term and multi term results for various transport properties for positrons in $\mathrm{N}_{2}$ when magnetic field of $500 \mathrm{Hx}$ is applied.

try of the electron velocity distribution function in velocity space follows. For electrons in $\mathrm{N}_{2}$, the two term approximation also fails [52], but the differences between the two term and fully converged transport data are much less pronounced. Interestingly, Ps formation appears to have little effect on the isotropy of the velocity distribution function. Similar observations have been made for attachment when electron transport comes into question [38]. For positrons, the anisotropy of the velocity distribution function is induced by an active role of inelastic collisions and hence the two term approximation generally fails in the energy range where the collision frequency for inelastic collisions becomes comparable to the collision frequency for elastic collisions.

The inadequacy of the two term approximation when a magnetic field is applied is illustrated in Figure 6. We see that the errors associated with the two term approximation are reduced comparing to an electric field only 
situation. Our careful analysis reveals that the general trend for the convergence in the $l$ index improves as $B / n_{0}$ increases. In other words, the accuracy of two term approximation is enhanced for an increasing $B / n_{0}$. Similar findings have been reported for the electrons [53-55].

\subsection{On validity of Langevin's elementary theory employed in positron transport studies}

It is shown in many classical textbooks $[29,56]$ that the electron mobility components parallel and perpendicular to the electric field are

$$
\begin{aligned}
& \mu_{\|}=\mu_{0}=\frac{e}{m \nu} \\
& \mu_{\perp}=\frac{1}{1+\Omega^{2}} \mu_{0}=\frac{e \nu / m}{\nu^{2}+\omega^{2}},
\end{aligned}
$$

where $\nu$ is the collision frequency for momentum transfer, $\omega$ is the cyclotron frequency, $\mu_{0}$ is the mobility for the magnetic field free case and $\Omega$ is the ratio between the collision and cyclotron frequencies. These equations follow from the simplified form of the momentum balance equation for light charged particles and are consistent with the so-called Langevin's elementary theory. For electrons, these equations have been often used in conjunction with the classical drift-diffusion equation and Maxwell's equation in fluid modeling of magnetized plasma discharges [57]. In the past, before sophisticated numerical solutions to Boltzmann's equation became available, equation (12) was extensively used in an inversion procedure for the determination of low-energy electron-molecule cross sections $[29,58]$. This formula can be later used to establish the diffusion coefficient for a magnetic field free case based on the Nernst-Townsend-Einstein relation

$$
D=\frac{W k_{B} T}{e E}=\frac{2}{3} \frac{W \epsilon}{e E},
$$

where $k_{B}$ is the Boltzmann constant, $e$ is the elementary charge, $W$ is the positron drift velocity and $\epsilon$ is the mean energy of charged particles. Notice that this formula holds for a Maxwellian distribution of charged particle velocities and does not distinguish between the longitudinal and transverse diffusion coefficients. When magnetic field is present, the diffusion coefficient for a charged particles in a direction normal to the magnetic field lines is usually scaled as

$$
D_{\perp}=\frac{D_{(B=0)}}{1+(\nu / \omega)^{2}},
$$

where $D_{(B=0)}$ is the magnetic field free diffusion coefficient and $D_{\perp}$ is the diffusion coefficient normal to a magnetic field. We should emphasize that this relation is incorrect on symmetry grounds - there is not an axis of rotational symmetry about the electric field direction $[13,53,54]$. We now wish to investigate the validity of these equations for positrons. Calculations are performed for positrons in $\mathrm{H}_{2}$ under the influence of electric and magnetic fields in a crossed field configuration. Similar program of investigation has been carried out in a comprehensive manner for electrons in certain model gases [14] and real gases $[17,55]$.

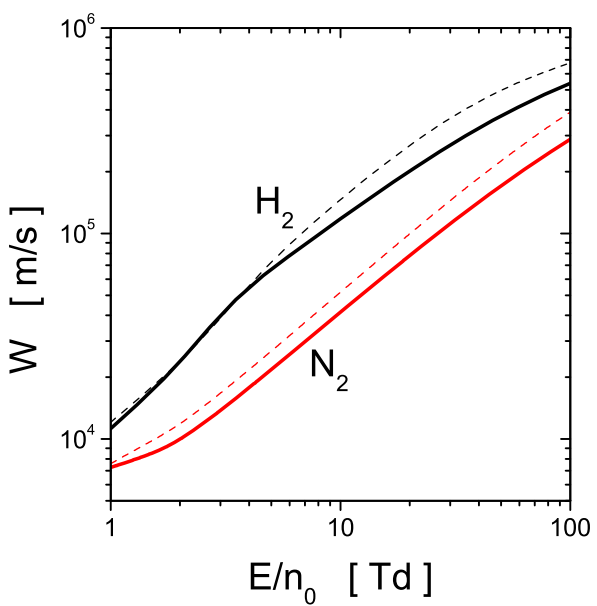

Fig. 7. (Color online) Variation of the drift velocity with $E / n_{0}$ for positrons in $\mathrm{H}_{2}$ and $\mathrm{N}_{2}$. Results obtained using a Langevin theory (dashed lines) are compared with those obtained by a multi term approach for solving Boltzmann's equation (full lines).

In Figure 7 we display the variation of the drift velocity with $E / n_{0}$ for positrons in $\mathrm{H}_{2}$ and $\mathrm{N}_{2}$. Results obtained by an elementary Langevin theory (Eq. (12)) are compared with highly accurate data calculated using a multi term approach for solving Boltzmann's equation. We observe that the Langevin theory generally tends to overestimate the accurate data. For electrons it was found empirically that it is more appropriate to use a modifying factor $F$ multiplying the right hand side of equation (12) [29,58]. From Figure 7 we see that the same strategy can be applied for positrons if one is forced to avoid the mathematical complexity associated with an accurate solution of Boltzmann's equation.

The Nersnt-Townsned-Einstein relation (Eq. (14)) for isotropic diffusion coefficient assuming Maxwell distribution of positron velocities is tested in Figure 8. We observe that the anisotropy of diffusion is considerable for both $\mathrm{H}_{2}$ and $\mathrm{N}_{2}$. Only in the limit of the lowest $E / n_{0}$ considered in this work, the diffusion of positrons in $\mathrm{H}_{2}$ becomes more isotropic in nature. This is a clear sign that any assumption of isotropic diffusion of positrons in molecular gases, e.g. $D_{L}=D_{T}=D$ can be in serious error.

The approximate expression for the diffusion coefficient of positrons normal to an electric field line given by equation (15) is tested in Figure 9. Comparison is made with the accurate data for the longitudinal diffusion coefficient and transverse diffusion coefficient along the $\boldsymbol{E} \times \boldsymbol{B}$ direction for positrons in $\mathrm{H}_{2}$. We see that the diffusion coefficients obtained by equation (15) and those obtained by a multi term theory for solving Boltzmann's equation are in poor agreement.

In Figure 10 we show the comparison between the Langevin theory (Eq. (13)) and accurate data for the $\boldsymbol{E} \times \boldsymbol{B}$ drift velocity component for $B / n_{0}$ of 100,200 and $2000 \mathrm{Hx}$. In the magnetic field controlled regime (for lower $E / n_{0}$, where $\omega \gg \nu$ ) the Langevin theory appears to be very inaccurate. However, as the system 


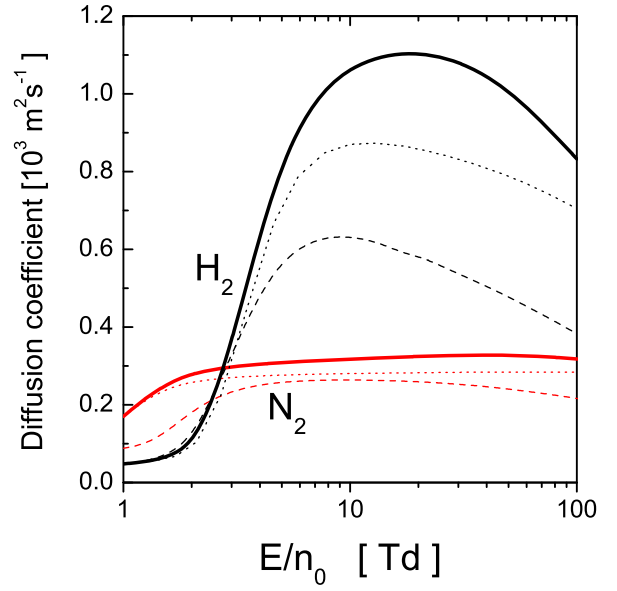

Fig. 8. (Color online) Variation of the diffusion coefficients with $E / n_{0}$ for positrons in $\mathrm{H}_{2}$ and $\mathrm{N}_{2}$. Longitudinal and transverse diffusion coefficients obtained by a multi term approach for solving the Boltzmann equation are represented with full and dashed curves, respectively, while the isotropic diffusion coefficients assuming the Maxwell distribution of velocities and Nernst-Townsend-Einsten relation are presented by doted curves.

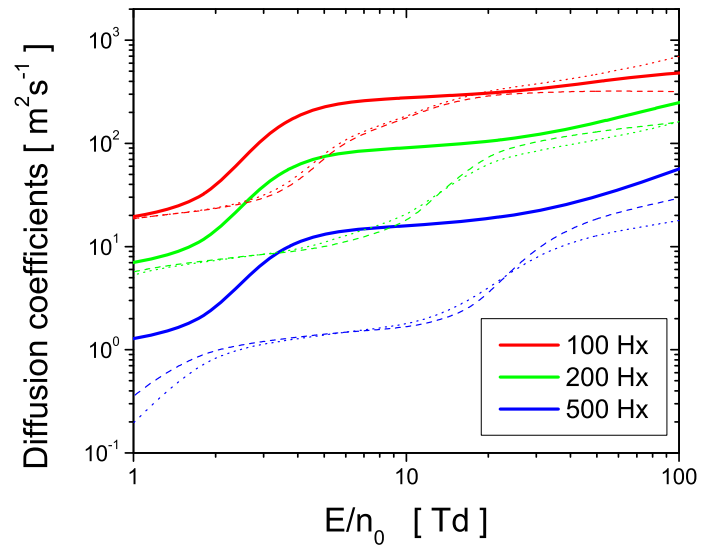

Fig. 9. (Color online) Variation of the diffusion coefficients with $E / n_{0}$ for positrons in $\mathrm{H}_{2}$. Longitudinal $D_{E}$ and transverse $D_{\boldsymbol{E} \times \boldsymbol{B}}$ diffusion coefficients obtained by a multi term approach for solving the Boltzmann equation are represented by dashed and dotted curves, respectively, while the scaled diffusion coefficient given by equation (15) is presented by the solid curve.

enters the collision dominated regime (higher $E / n_{0}$, where at first $\nu \approx \omega$, and then $\nu \gg \omega)$ the accuracy of the Langevin theory is improved. Substantial increase in accuracy of Langevin's theory could be achieved by including an energy dependent collision frequency. Apart from a multi term theory for solving Boltzmann's equation and Monte Carlo simulation technique, a good candidate for the provision of such relations is momentum transfer theory $[34,59,60]$. Therefore, one should bear in mind the inherent assumptions associated with the Langevin theory and remember that the obtained results will be of limited accuracy for positrons, particularly under conditions when the magnetic field controls the motion of positrons.

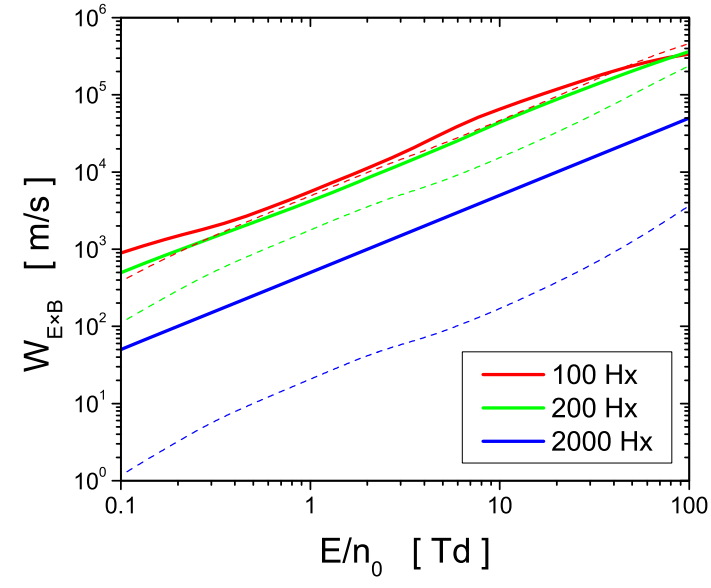

Fig. 10. (Color online) Variation of the transverse drift velocity component along the $\boldsymbol{E} \times \boldsymbol{B}$ direction with $E / n_{0}$ and $B / n_{0}$ (full lines: multi term Boltzmann equation results; dashed lines: Langevin theory results).

\subsection{How accurate is Tonks' theorem for positrons?}

In this section we explore the validity of Tonks' theorem for positrons in electric and magnetic fields. The motivation behind employing Tonks' theorem is that one may attempt to use the positron transport data obtained from electric field only calculations to the more general case involving both the electric and magnetic fields [30-34]. However, Tonks' theorem has a restricted domain of application on the mean energy, drift speed and rate coefficients. In other words, this approximation cannot be used to calculate the individual elements of the drift velocity and diffusion tensor.

Tonks' theorem falls into category of effective field approximations and it assumes that the average energy and drift speed are, respectively, given by $[33,34]$

$$
\begin{aligned}
\epsilon(E, B, \phi) & =\epsilon\left(E_{\mathrm{eff}}, 0,0\right), \\
W(E, B, \phi) & =\left(E_{\mathrm{eff}}, 0,0\right),
\end{aligned}
$$

where $E_{\text {eff }}$ is an effective field whose magnitude is given by

$$
E_{\text {eff }}(\epsilon)=E \sqrt{\frac{1+\left(\omega / \nu_{m}\right)^{2} \cos ^{2} \phi}{1+\left(\omega / \nu_{m}\right)^{2}}} .
$$

Here $\omega$ denotes the cyclotron frequency of the positrons while $\nu_{m}$ is the average momentum transfer collision frequency evaluated at $E$, and $\phi$ is the angle between the electric and magnetic fields. Equations (16)-(18) represent a system of non-linear equations which has been solved iteratively.

In Figures 11-13 we show a comparison between the mean energies, rate coefficients for Ps formation and flux drift velocity for positrons in $\mathrm{N}_{2}$ obtained by Tonks' theorem and an accurate multi term approach for solving Boltzmann's equation. Among many interesting points, the following are of particular importance: (i) Tonks' theorem generally tends to overestimate the actual value of the mean energy and to underestimate the actual value 


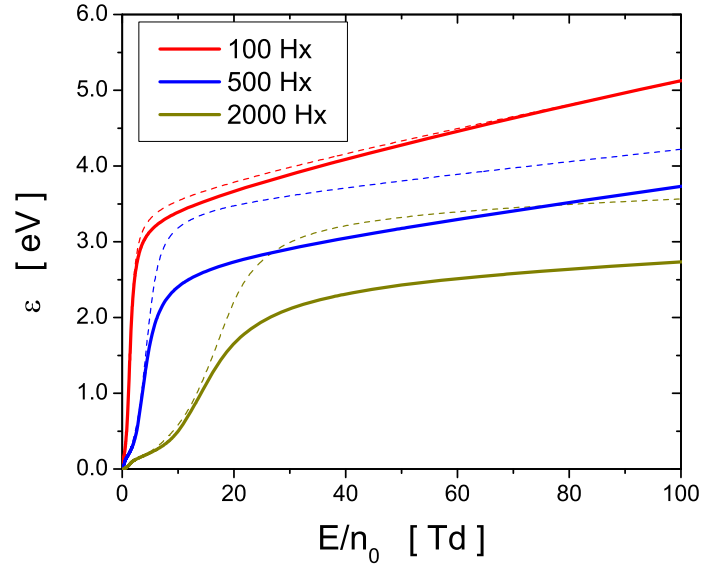

Fig. 11. (Color online) Variation of the mean energy with $E / n_{0}$ and $B / n_{0}$ in a crossed field configuration for positrons in $\mathrm{N}_{2}$. Exact data obtained by a multi term approach for solving Boltzmann's equation are presented by solid lines while Tonks' theorem results are presented by dashed lines.

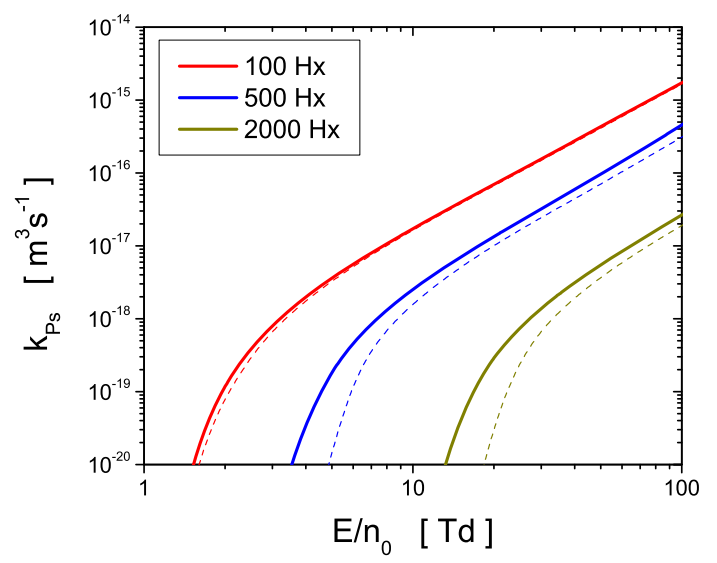

Fig. 12. (Color online) Variation of the rate coefficient for Ps formation with $E / n_{0}$ and $B / n_{0}$ in a crossed field configuration for positrons in $\mathrm{N}_{2}$ for the same conditions as those in Figure 11.

of rate coefficient for Ps formation; (ii) Tonks' theorem increases in accuracy as the system enters the collision dominated regime; and (iii) for an increasing $B / n_{0}$ the accuracy of Tonks' theorem is deteriorated. The quantitative differences between the accurate data and those obtained by Tonks' theorem are mainly due to the variation of the collision frequency and distribution function with $B / n_{0}$. For those values of $E / n_{0}$ where the averaged momentum transfer collision frequency exhibits a weak sensitivity with respect to the reduced magnetic field, the accuracy of Tonks' theorem is increased.

In Figures 14 and 15 we compare the results for the bulk drift velocity components in $\mathrm{N}_{2}$ and $\mathrm{H}_{2}$, respectively, obtained by Tonks' theorem and an accurate multi term theory for solving Boltzmann's equation in a crossed field configuration. We can see that the agreement is not so good as for the flux components shown in Figure 13, particularly for positrons in $\mathrm{H}_{2}$, where strong disagreement between the accurate data and those obtained by Tonks'

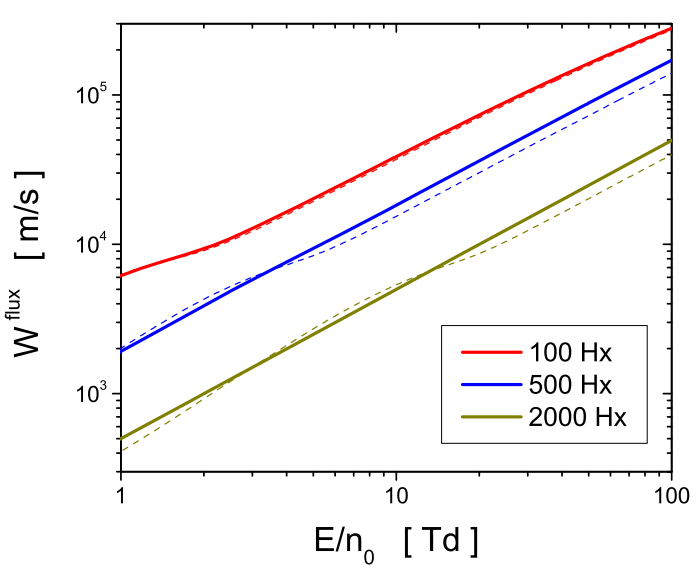

Fig. 13. (Color online) Variation of the flux drift velocity component with $E / n_{0}$ and $B / n_{0}$ in a crossed field configuration for positrons in $\mathrm{N}_{2}$ for the same conditions as those in Figure 11.

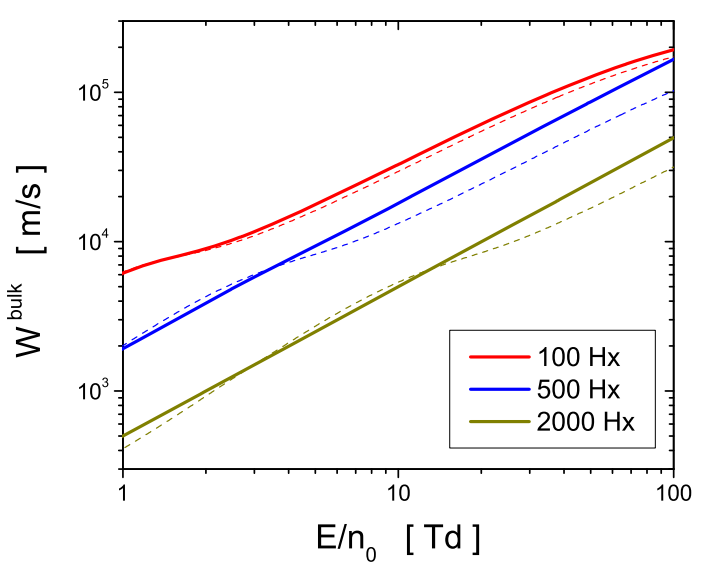

Fig. 14. (Color online) Variation of the bulk drift velocity component with $E / n_{0}$ and $B / n_{0}$ in a crossed field configuration for positrons in $\mathrm{N}_{2}$ for the same conditions as those in Figure 11.

theorem is clearly evident. We observe a very pronounced NDC effect in the profiles of the bulk drift velocity for $B / n_{0}$ of 100 and $500 \mathrm{Hx}$ calculated by Tonks' theorem. On the other hand, from accurate data we see that NDC is absent when magnetic field is applied. This suggests that if Ps formation is a dominant process comparing to other inelastic channels, then the results for the bulk drift velocity component obtained by Tonks' theorem may not be even qualitatively correct. Similar behavior has been recently found for positrons in water vapor [47]. For positrons in $\mathrm{N}_{2}$, however, the situation is not so dramatic although the quantitative differences between the accurate data and those obtained by Tonks' theorem are considerable. In conclusion, Tonks' theorem fails to adequately describe the bulk positron drift in molecular gases properly under the conditions considered in this work. 


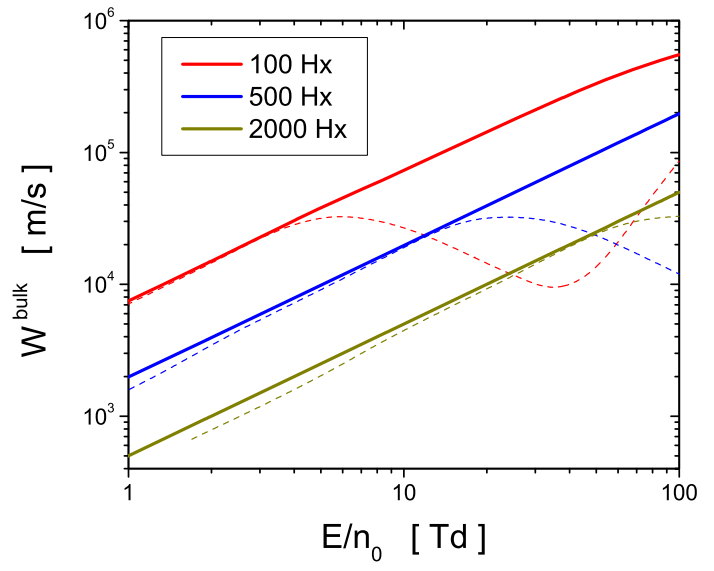

Fig. 15. (Color online) Variation of the bulk drift velocity component with $E / n_{0}$ and $B / n_{0}$ in a crossed field configuration for positrons in $\mathrm{H}_{2}$ for the same conditions as those in Figure 11.

\section{Conclusion}

Using a multi term solution of Boltzmann's equation, we have tested various approximations for the determination of positron transport properties in neutral gases in crossed electric and magnetic fields. We have (1) tested the approximation of using the cross sections for the electron scattering to describe the positron behavior; (2) demonstrated the differences between the results for various transport properties obtained by the two-term approximation and multi term theory for solving Boltzmann's equation; (3) illustrated the range of applicability of Langevin's elementary theory and Tonks' theorem for positrons in crossed electric and magnetic fields. Included in the discussion are NDC phenomena, the duality of the drift velocity and diffusion tensor transport coefficients due to the explicit effects of Ps formation and the anisotropy of the diffusion tensor for positrons in $\mathrm{N}_{2}$ and $\mathrm{H}_{2}$.

It is found that approximations based on using the cross sections for the electron scattering to describe positron behavior can seriously compromise the accuracy of modeling positron transport. The inadequacy of such assumptions is demonstrated through the differences between the $E / n_{0}$ profiles of the mean energy, drift velocity and diffusion coefficients for positrons and electrons. In particular, it is shown that the flux and bulk transport properties can vary substantially from one another for both the electrons and positrons. While for electrons the mismatch between these two sets of data is only of a small quantitative difference only, for positrons the situation is more dramatic. A very pronounced NDC effect has been observed in the bulk drift velocity for positrons in $\mathrm{H}_{2}$, a phenomenon which is absent from the profiles of the drift velocity components for electrons in $\mathrm{H}_{2}$. This clearly reflects the differences in the basic phenomenology between the transport of positrons and electrons in the same molecular gas.

For the accurate calculation of positron transport properties in molecular gases under the influence of electric and magnetic fields, it appears that a multi term approach for solving Boltzmann's equation and/or Monte Carlo simulation technique is inevitable. The errors associated with the two term approximation are highlighted, including those data which are most sensitive to the approximation. It is found that the magnetic field reduces the asymmetry of the distribution function in velocity space and thus improves the accuracy of the two term approximation. The Langevin theory results for the drift velocity components are also of limited accuracy, particularly in crossed electric and magnetic fields for conditions when the cyclotron frequency dominates the collision frequency. It is shown that the mismatch between the Langevin theory and accurate results sensitively depends on the magnitude of the cross sections of the various positron-molecule collision processes involved.

A caution is issued in this paper on how to employ Tonks' theorem for positrons in electric and magnetic fields. Large discrepancies between Tonks' theorem and accurate results are found for the bulk drift velocity of positrons in $\mathrm{H}_{2}$, reflecting that Tonks' theorem cannot handle the explicit contribution of Ps formation on the distribution function and the corresponding effects on the drift.

Authors are grateful to J. Marler, G. Malović, J. Sullivan and C. Surko for discussion on different sources of data. This work was supported by MNRS Projects ON171037 and III41011, and Australian Research Council. SD acknowledges also support from STW-project 10118, part of the Netherlands Organization for Scientific Research (NWO).

\section{References}

1. M. Charlton, J. Humberston, Positron Physics (Cambridge University Press, New York, 2000)

2. Z.Lj. Petrović, A. Banković, S. Dujko, S. Marjanović, M. Šuvakov, G. Malović, J.P. Marler, S.J. Buckman, R.D. White, R.E. Robson, J. Phys.: Conf. Ser. 199, 012016 (2010)

3. Z.Lj. Petrović, A. Banković, S. Marjanović, M. Šuvakov, S. Dujko, G. Malović, R.D. White, S.J. Buckman, J. Phys.: Conf. Ser. 262, 012046 (2011)

4. N. Guessoum, P. Jean, W. Gillard, Mon. Not. R. Astron. Soc. 42, 1171 (2010)

5. G.F. Gribakin, J.A. Young, C.M. Surko, Rev. Mod. Phys. 82, 2557 (2010)

6. R.L. Wahl, Principles and Practice of Positron Emission Tomography (Lippincott, Williams and Wilkins, Philadelphia, 2002)

7. S.R. Cherry, J.A. Sorensen, M.E. Phelps, Physics in Nuclear Medicine (Saunders, Philadelphia, 2003)

8. R.D. White, R.E. Robson, Phys. Rev. Lett. 102, 230602 (2009)

9. J.P. Marler, C.M. Surko, Phys. Rev. A 72, 062702 (2005)

10. S. Marjanović, M. Šuvakov, A. Banković, M. Savić, G. Malović, S.J. Buckman, Z.Lj. Petrović, IEEE Trans. Plasma Sci. 39, 2614 (2011)

11. C.M. Surko, G.F. Gribakin, S.J. Buckman, J. Phys. B: At. Mol. Opt. Phys. 38, R57 (2005)

12. J.P. Sullivan, C. Makochekanwa, A. Jones, P. Caradonna, S.J. Buckman, J. Phys. B: At. Mol. Opt. Phys. 41, 081001 (2008) 
13. R.D. White, R.E. Robson, S. Dujko, P. Nicoletopoulos, B. Li, J. Phys. D 42, 194001 (2009)

14. S. Dujko, R.D. White, Z.Lj. Petrović, R.E. Robson, Phys. Rev. E 81, 046403 (2010)

15. Z.M. Raspopović, S. Sakadzić, S. Bzenić, IEEE Trans. Plasma Sci. 27, 1241 (1999)

16. S. Dujko, Z.M. Raspopović, Z.Lj. Petrović, J. Phys. D 38, $2952(2005)$

17. S. Dujko, R.D. White, K.F. Ness, Z.Lj. Petrović, R.E. Robson, J. Phys. D 39, 4788 (2006)

18. M. Šuvakov, Z.Lj. Petrović, J.P. Marler, S.J. Buckman, R.E. Robson, G. Malović, New J. Phys. 10, 053034 (2008)

19. J.P. Marler, Z.Lj. Petrović, A. Banković, S. Dujko, M. Šuvakov, G. Malović, S.J. Buckman, Phys. Plasmas 16, 057101 (2009)

20. I. Kawrakow, Med. Phys. 27, 485 (2000)

21. F.B. Brown, MCNP-a general Monte Carlo N-particle transport code, Version 5 Report LA-UR-03-1987 (Los Alamos, NM, Los Alamos National Laboratory, 2003)

22. S. Agostinelli et al., Nucl. Instrum. Methods A 506, 250 (2003)

23. J. Baró, J. Sempau, J.M. Fernandez-Varea, F. Salvat, Nucl. Instrum. Methods B 100, 31 (1995)

24. H. Zaidi, Med. Phys. 26, 574 (1999)

25. G. Garcia, Z.Lj. Petrović, R.D. White, S.J. Buckman, IEEE Trans. Plasma Sci. 39, 2962 (2011)

26. R.E. Robson, P. Nicoletopoulos, B. Li, R.D. White, Plasma Sources Sci. Technol. 17, 024020 (2008)

27. R.E. Robson, R.D. White, Z.Lj. Petrović, Rev. Mod. Phys. 77, 1303 (2005)

28. E.H. Holt, R.E. Haskell, Foundations of Plasma Dynamics (The Macmillan Company, New York, 1965)

29. L.G.H. Huxley, R.W. Crompton, Drift and Diffusion of Electrons in Gases (Wiley-Interscience, New York, 1974)

30. L. Tonks, Phys. Rev. 51, 744 (1937)

31. L. Tonks, W.P. Allis, Phys. Rev. 52, 710 (1937)

32. A.E.D. Heylen, IEE Proc. 127, 221 (1980)

33. R.D. White, R.E. Robson, K.F. Ness, IEEE Trans. Plasma Sci. 27, 1249 (1999)

34. R.E. Robson, Aust. J. Phys. 47, 279 (1994)

35. L. Boltzmann, Wein. Ber. 66, 275 (1872)

36. C.S. Wang-Chang, G.E. Uhlenbeck, J. DeBoer, in Studies in Statistical Mechanics, edited by J. DeBoer, G.E. Uhlenbeck (Wiley, New York, 1964), Vol. 2, p. 241
37. R.E. Robson, K.F. Ness, Phys. Rev. A 33, 2086 (1986)

38. K.F. Ness, R.E. Robson, Phys. Rev. A 34, 2185 (1986)

39. R.D. White, K.F. Ness, R.E. Robson, Appl. Surf. Sci. 192, $26(2002)$

40. K. Kumar, H.R. Skullerud, R.E. Robson, Aust. J. Phys. 33, 343, (1980)

41. V.D. Stojanović, Z.Lj. Petrović, J. Phys. D 31, 834 (1998)

42. Jung-Sik Yoon, Mi-Young Song, Jeong-Min Han, Sung Ha Hwang, Won-Seok Chang, BongJu Lee, Yukikazu Itikawa, J. Phys. Chem. Ref. Data 37, 913 (2008)

43. R.W. Crompton, Adv. At. Mol. Opt. Phys. 32, 97 (1994)

44. A. Banković, J.P. Marler, M. Šuvakov, G. Malović, Z.Lj. Petrović, Nucl. Instrum. Methods B 266, 462 (2008)

45. A. Banković, S. Dujko, R.D. White, S. Buckman, Z.Lj. Petrović, Nucl. Instrum. Methods B. 279, 92 (2012)

46. A. Banković, Z.Lj. Petrović, R.E. Robson, J.P. Marler, S. Dujko, G. Malović, Nucl. Instrum. Methods B 267, 350 (2009)

47. A. Banković, S. Dujko, R.D. White, J.P. Marler, S.J. Buckman, Z.Lj. Petrović, New J. Phys. 14, 035003 (2012)

48. N. Bose, D.A. Paul, J.S. Tsai, J. Phys. B: At. Mol. Phys. 14, L227 (1981)

49. M. Charlton, J. Phys. B: At. Mol. Phys. 18, L667 (1985)

50. N.A. Dyatko, A.P. Napartovich, S. Sakadžić, Z. Petrović, Z. Raspopović, J. Phys. D 33, 375 (2000)

51. S. Dujko, Z.M. Raspopović, Z.Lj. Petrović, T. Makabe, IEEE Trans. Plasma Sci. 31, 711 (2003)

52. S. Dujko, U. Ebert, R.D. White, Z.Lj. Petrović, Jpn J. Appl. Phys. 50, 08JC01 (2011)

53. K.F. Ness, J. Phys. D 27, 1848 (1994)

54. R.D. White, K.F. Ness, R.E. Robson, B. Li, Phys. Rev. E 60, 2231 (1999)

55. S. Dujko, R.D. White, Z.Lj. Petrović, R.E. Robson, Plasma Sources Sci. Technol. 20, 024013 (2011)

56. M.A. Lieberman, A.J. Lichtenberg, Principles of Plasma Discharges and Materials Processing (Wiley-Intersciences, Hoboken NJ, 2005)

57. G.J.M. Hagelaar, Plasma Source Sci. Technol. 16, S57S66 (2007)

58. Z.Lj. Petrović, S. Dujko, D. Marić, G. Malović, Ž Nikitović, O. Šašić, J. Jovanović, V. Stojanović, M. RadmilovićRadjenović, J. Phys. D 42, 194002 (2009)

59. R.E. Robson, J. Chem. Phys. 85, 4486 (1986)

60. S.B. Vrhovac, Z.Lj. Petrović, Phys. Rev. E 53, 4012 (1996) 